\title{
Risk Assessment Approach in Airport Security
}

\author{
Mahardi Sadono $^{1)^{*}}$, Saphira Alifa Harahap ${ }^{1)}$, Dani Yulianto Putra ${ }^{1)}$, Randhy ${ }^{1)}$, Muhamad Luthfi Imam Nurhakim ${ }^{1)}$
}

\begin{abstract}
Naturally, the threat to flight is generated from all activities at the airport by passenger or freight. With the growth of air transport, the level of threat to flight activity will also increase. Due to ensure the security of passengers, staff, and all elements of the airport, the airport as a place of the flight operations need a reliable protection system. The purpose of this study is to develop a methodology for assessing the risk level at the airport in order to improve the level of protection at the airport. The level of threats that could jeopardize airport security is categorized into several levels, then analyzed to what extent the risk level can be tolerated as a reference to the formulation of standard protection systems at the airport. The results of this study can be used to deal with threats that occur in the airport through preventive action by setting up a reliable protection system. The risk assessment approach also able to evaluate the airport's existing protection system whether it is tolerable to deal with threats or not, so action can be taken to improve the protection system if the failure rate is still high and have massive consequences to the all airport elements.
\end{abstract}

Keywords - airport security, failure rate, protection system, risk assessment, tolerable risk level.

\section{INTRODUCTION}

\section{A. Airport Security}

I

$\mathrm{n}$ this era, the requirement of air transportation which can saving more time than other transportation type is increasing. This can be seen from growth rate of airplane movement and increased number of passengers in Indonesia [1]. It is related to airport security. Airport security is a system of methods and technology that used to protect all elements in the airport from the threats.

On several occasions, Indonesian airport encountered threats that can ruin airport facility, and also threatening safety of passengers and staffs. Last case occurred in Sam Ratulangi Airport when some people break through the airport and causing the glass of arrival gate broken. It shows the importance of airport security to ensure the safety of the passengers, staff, and all elements of the airport.

\section{B. Previous Research Results}

Security issues always have been a problem for aviation industry and organization, and it always increase over years.

${ }^{1)}$ Faculty of Mechanical and Aerospace Engineering, Institut Teknologi Bandung, Indonesia. ${ }^{*}$ mahar65@yahoo.com
Several researches have been conducted to provide some recommendations to solve problems occurred in aviation history, as well as to prevent potential problems that might happened in the future. Previous research showed that sets of scenario towards the specific target can be investigated through examination of its vulnerability and its criticality [2]. Every potential scenario has been studied in order to have an estimation of the current risk level. The quantitative analysis is possible to investigate the vulnerabilities and criticality of the airport components. The optimized design of the airport security, its ability to innovate, and to modify itself in consequence of the results of risk assessment, is the best indicator of the ability to answer new threats and to assure an acceptable security risk level. The approached is shown in the Figure 1.

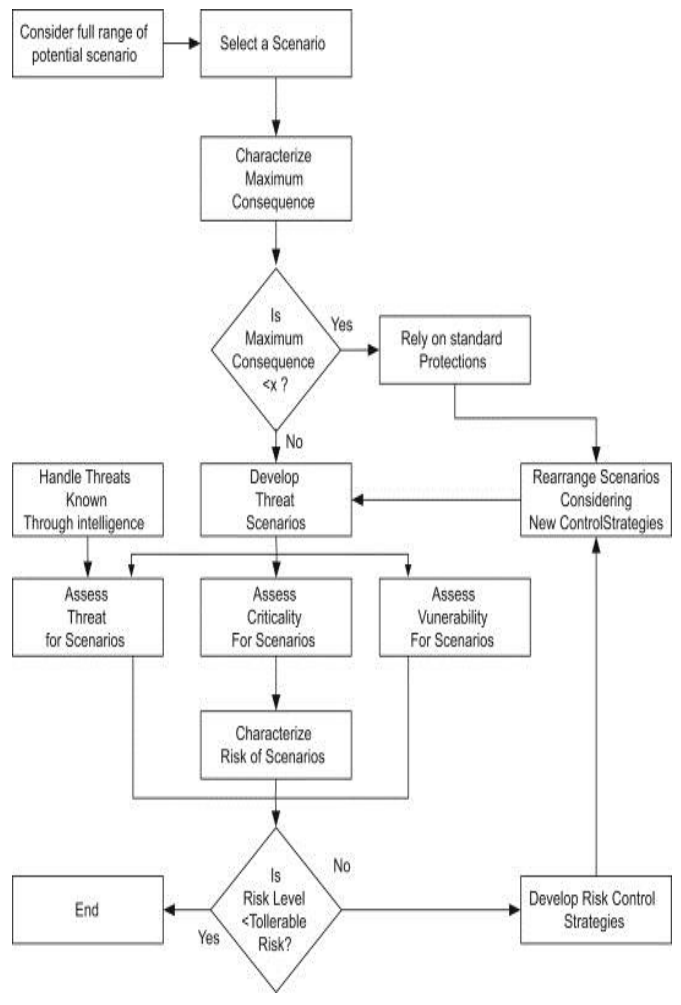

Fig 1. Risk assessment approach [2]

In order to categorizing the risk level, another research was conducted specifically for Indonesian Airports. The cases have been categorized into 4 risk levels [3]. This study 
use six parameters to categorize the risk level: airport location, VIP flight, apron capacity, number of runway exist, international routes, and air traffic. Each parameters will give a score (range: 1-5) and the total score of these parameters will be used to categorize the risk level. The $1^{\text {st }}$ level is the airport with the highest risk with score greater than 36 , and the lowest risk will be the $4^{\text {th }}$ level that have score less than 17. The work then analyzed 172 airports in Indonesia and categorized them into 4 level of risk based on the method.

A research program [4] which was conducted by Airport Cooperation Research Program (ACRP) sponsored by FAA released a guidebook for safety management for Airports in 2015 to help airports organization to prevent any security violation that might harming passengers or causing financial losses and as a guidance for airport operators, local agencies, the FAA, and other interested parties, and industry associations may arrange for workshops, training aids, field visits, and other activities. This research project is assigned to an expert panel, appointed by the Transportation Research Board (TRB). Panels include experienced practitioners and research specialists; heavy emphasis is placed on including airport professionals, the intended users of the research products. On this research the panels prepare project statements (requests for proposals), select contractors, and provide technical guidance and counsel throughout the life of the project. The process for developing research problem statements and selecting research agencies has been used by TRB in managing cooperative research programs since 1962. As in other TRB activities, ACRP project panels served voluntarily without compensation. This research provide guidance on conducting the Safety Risk Management (SRM) process, one of the four components of the Safety Management System (SMS) is shown in Figure 2.

\section{Regulation of Airport Security}

There are some regulations that regulate about Airport Security. Locally, Indonesia has PM 31 year 2013[5]: National Aviation Security Program (Program Keamanan Penerbangan Nasional) that contain regulations and have some objectives to protect security, safety, regularity, and efficiency of Indonesia's Aviation. PM 31 year 2013 also regulate about Standard and Procedure that has to be complied by passengers, aviation crew, aviation security crews, and also citizen to prevent acts of unlawful interference. For international regulations, there are ICAO Doc 8973/8 about Aviation Security Manual [6].

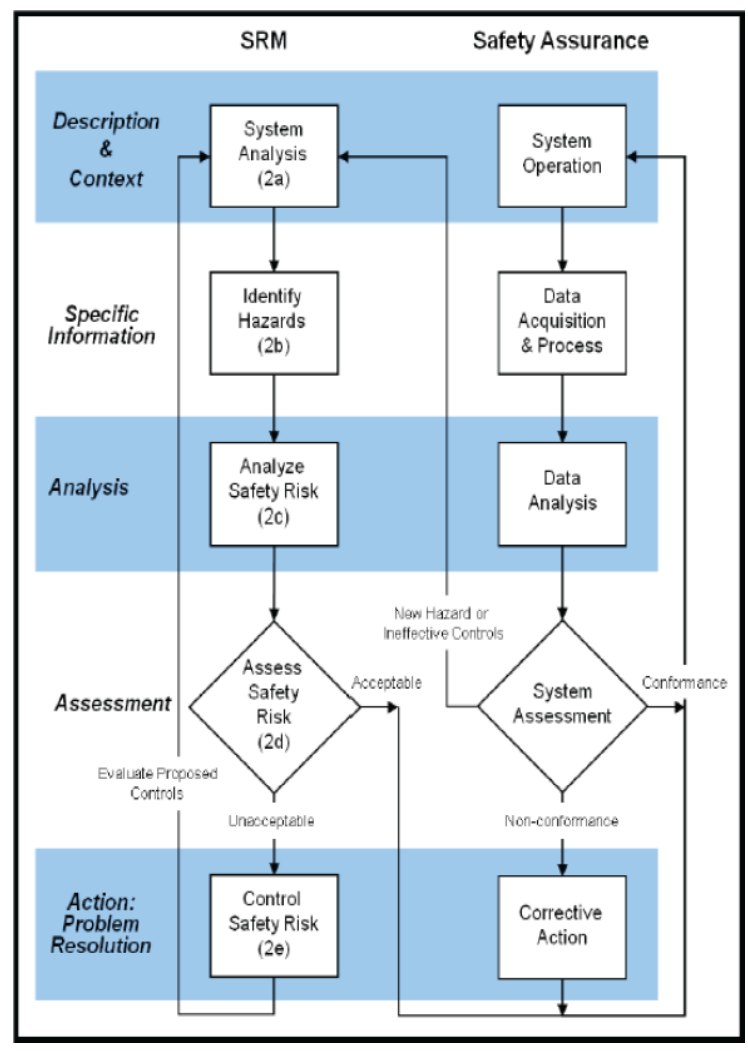

Fig 2. SRM and safety assurance processes (FAA Order 8040.4A, 2012)

ICAO Doc 8973/8 has an objective to advise States on the prevention of and where necessary response to act of unlawful interference, through the application of an aviation security system comprised of four main elements: legal framework and security oversight; airport design, infrastructure and equipment; human resources recruitment, selection, training and certification; and procedures and the implementation of security measures.

\section{METHODOLOGY}

This research has an objective to make a risk assessment approach which will more efficient than the previous researches. There are three questions that need to be explained to reach the objective:

1) How to make Risk Assessment Approach?

2) How to categorize the threats of Airport Security? and,

3) How to determine a tolerable risks level?

To occupy these questions, this research continues the previous research in 2013 entitled "Study of Indonesian Airports Risk Level Categories". Based on that research we 
adopt the level risk method to categorize threats level that will be faced. This research also refers to "Risk Assessment Techniques for Civil Aviation Security" from Porto Institutional Repository and "A guidebook for Safety Risk Management for Airports" from ACRP report 131 to be another consideration to compile the method in this research. The research process can be seen in the Figure 3 below.

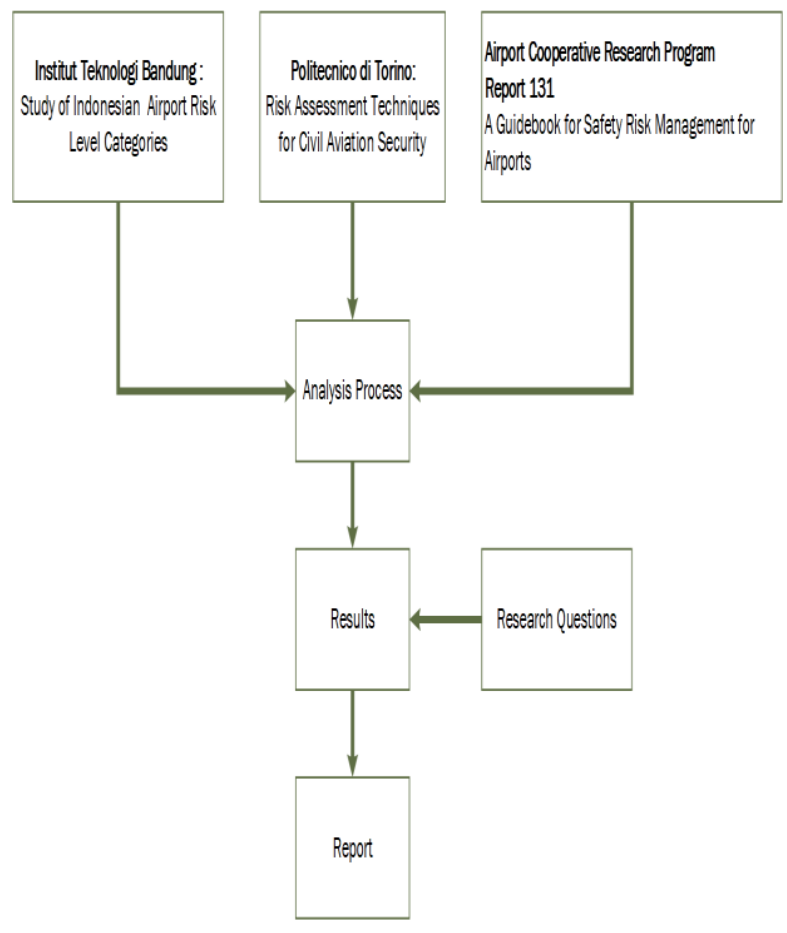

Fig 3. Research Process

According to Business Dictionary, risk is a probability or threat of damage, injury, liability, loss, or any other negative occurrence that is caused by external or internal vulnerabilities, and that may be avoided through preemptive action. While threat is a negative event, it can cause a risk to become a loss, expressed as an aggregate of risk, consequences of risk, and the likelihood of the occurrence of the event. Threats can also come from the many processes that support an airport and the passengers and aircraft it serves: catering, maintenance, cleaning, ticketing, baggage handling, air traffic control, retail, food services, parking, car rental and others.

There are many risk tool management that can be used to assess the problems. Fault Tree analysis was chosen as risk tool management to analyze risks in this research. Fault Tree analysis is a top down, deductive failure analysis to understand of how an undesired event can happen. This method identifies the best ways to reduce risk or to determine of how many times of a safety accident or particular system fails. Fault Tree Analysis helps to identify the potential cause of a problem before it actually occur. Fault Tree Analysis can also be used to evaluate the probability of a system failure using analytical or statistical method.

\section{ANALYSIS}

\section{A. Risk Assessment Approach}

In previous research held by ITB [3], the research only focus on the threat analysis without adding protection system on its risk assessment approach. On the other research conducted by Politecnica di Torino [2], their risk assessment approach already considering the scenario that may happen and also considering the scenario's likelihood and consequences. However, it has not divided the risk level on three levels (low, med, and high), it only has two risk level (low and high). The Risk Assessment Approach will be started with threat which possible to happened in the Airport. With this condition, listing down the scenario that may happen that will lead to threat for airport security is required. There are some acts that avowed as Acts of Unlawful Interference (PM 31 year 2013):

1) Take over airplane's control,

2) Destroy airplane in land,

3) Holding hostage in airplane or airport,

4) Forced entry to airplane, airport or aeronautics place,

5) Bring weapon or dangerous goods,

6) Using airplane outside the procedure,

7) Give the wrong information, and

8) Bomb threats

These acts of unlawful interference will lead to some scenario that might happen and will threaten the airport security, it can be seen in Figure 2. The scenarios then will be analyzed to get the result whether the scenario has low risk or high risk on airport security. If the risk is low, so the scenario will be ignored. But if the risk is high, the airport management should have prepared protection standard against the threat so the scenario will not happen. If the airport has no protection standard to prevent the scenario, the airport needs to develop protection system to protect its security.

If the airport already had protection standard, the next step is to analyze the probability of the protection system failure. If the result from the analyzation process is 
categorized as low rate failure, the airport security already has adequate airport security system. This result is the ideal condition for the airport security system, because it means that the probability of the threat to happen is low. If the failure rate is categorized as medium, then they will need to make small improvement in the protection system. The improvement could be applied on the software, hardware, or the liveware of security system. After the improvement applied, the system failures need to be checked again, is it already have low failure rate or not. If not, so there will be need more improvements until the failure rate is low. Last, if the failure rate is high, then the airport needs to develop protection system. It will have same steps as the airport's condition when the airport doesn't have any protection standard. New or developed protection system needs to be analyzed whether the system decreases the failure rate to acceptable amount. If the failure rate hasn't reach the acceptable amount, then the airport needs make new protection system or develop existing protection to decrease the failure rate.

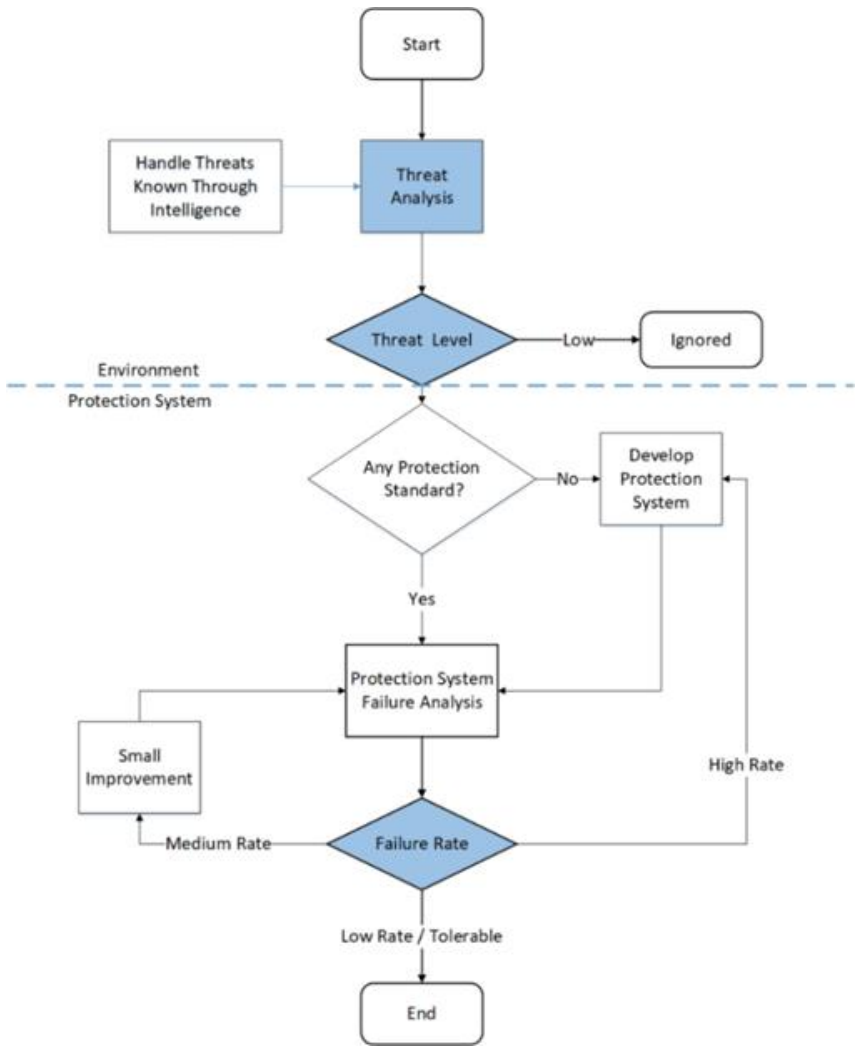

Fig 4. Airport Risk Assessment approach flow process

\section{B. Threat Category on Airport Security}

If we break down the list of scenarios that might happen and cause threats to Airport Security, there will be many. The question is, is it necessary to make protection to every threats ahead? To answer that question in ideal condition is yes. But, that will need much works, times, and of course money. So that, categorizing which threats' scenario that needs security protection is required.

The threat could be categorized by it likelihoods and consequences the threat will happen in the airport. There are needs to select the scenario that might happen that will make acts of unlawful interferences [2]. The scenarios selected are the threats that needed the protection system. If the threats have high likelihood or high consequences, so the threat categorized as threats that will make acts of unlawful interference, so that needed protection system. If the threats have low consequences and low likelihood, there will be no need to make protection standard. Table 1 shows the matrix of threat category.

Table 1. Threat Category on Airport Security Matrix

\begin{tabular}{c|c|c}
\hline & $\begin{array}{c}\text { Low } \\
\text { Likelihood } \\
\text { (LL) }\end{array}$ & $\begin{array}{c}\text { High } \\
\text { Likelihood } \\
\text { (HL) }\end{array}$ \\
\hline $\begin{array}{c}\text { Low } \\
\text { Consequences } \\
\text { (LC) }\end{array}$ & (a) & (b) \\
\hline $\begin{array}{c}\text { High } \\
\text { Consequences } \\
\text { (HC) }\end{array}$ & (c) & (d) \\
\hline
\end{tabular}

Explanation from Table 1:

(a): Low Consequences and Low Likelihood, so it safe

(b): Low Consequences and High Likelihood, because there are High Likelihood in this condition so it would be proceeded to the next procedure

(c): High Consequences and Low Likelihood, because there are High Consequent in this condition so it would be proceeded to the next procedure

(d): High Consequences and High Likelihood, because both are in high condition so it would be proceeded to the next procedure

Tolerable risk level is the value that could be passed as good protection standard for airport security, so there will be no need to make improvement on protection standard exist. In 
aviation safety, there are safety risk level that categorize the probability of failure happened in aviation as seen in Figure 5. This safety risk levels will be used as reference on deciding tolerable risk level on airport security. Variable used on deciding the level risk is the failure rate of the protection system. There are three levels of tolerable risks level:

- Low failure rate, where system's failure rate less than $10^{-5}$

- Medium failure rate, where system's failure rate between $10^{-3}$ and $10^{-5}$

- High failure rate, where system's failure rate bigger than $10^{-3}$

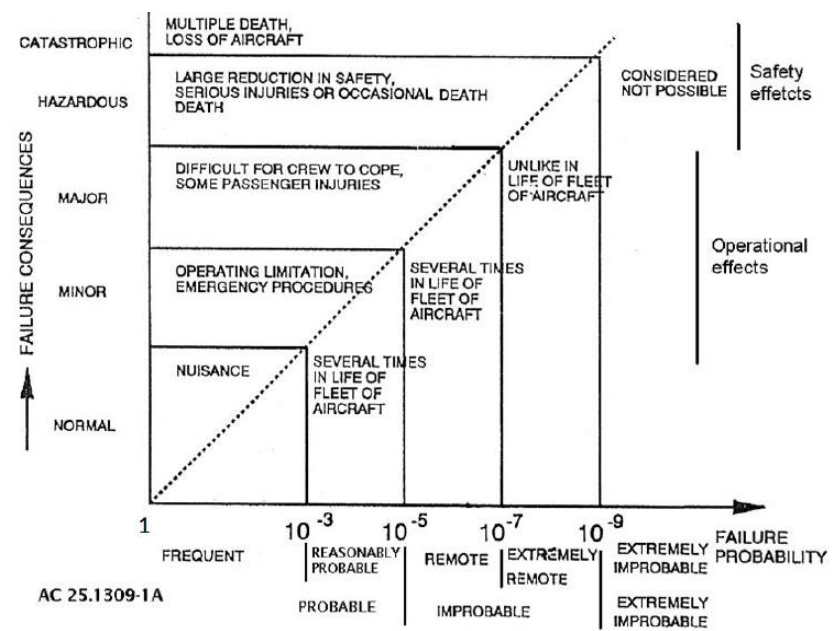

Fig 5. Aviation Safety Risks Level

The system's failure rate could be obtained by using Fault Tree Analysis Methods. This method will give value of system's failure rate. After the value obtained, the acts further that will be applied on the system can be decide. If the failure rate is low, so there no need acts applied on the protection system. If the failure rate is medium, so there will be need some improvement on protection system, whether its software, hardware, or live ware. And if the failure rate is high, there will be need to the airport to develop its protection system.

\section{RESULTS}

The airport risk assessment approach resulting from this research is the process that starting from threats analysis by using the likelihood and consequences of the scenario that could make a threat to airport security. If the result has a high likelihood or high consequences, so the next step of the process is analyze the airport's protection system. But if the result is the scenario will have a low likelihood chances and low consequences, so the scenario could be ignored.

If the airport has no protection standard, so there will be needed to develop protection system on the airport. But if the airport already has protection standard, the next thing to do is analyze the protection system failure rate. The tools will be used on this analysis is fault tree analysis methods. The result of fault tree analysis will be standard to decide on its failure rate level. Is it high rate level, medium rate level, or low rate level? The good protection system is the system with low failure rate. So, if the system's failure rate is medium there will be need small improvement of protection system. If the failure rate is high, so there will be need to redevelop protection system.

This research completed the late research $[2,3]$ on having a process to select threats exist that need protection system or not using threat category on airport security matrix. Besides that, this research also give a method recommendation on failure rate analysis of the protection system, which is Fault Tree Analysis. The risk level also given by the reference of aviation safety risk level.

\section{REFERENCES}

[1] Statistical Handbook of Indonesia. Biro Pusat Statistik, 2016.

[2] Tamasi G., Demichela M. Risk Assessment Techniques for Civil Aviation Security. 2011.

[3] Nurhakim, M.L.I.N., Sadono, M., and Chandra, D. Study of Indonesian Airports Risk Level Categories. $5^{\text {th }}$ Regional Conference on Mechanical and Aerospace Technology, Bangkok, February 2013.

[4] Airport Cooperative Research Program. Federal Aviation Administration, 2015.

[5] Program Keamanan Penerbangan Nasional (National Aviation Security Program). PM 31 Tahun 2013, Indonesia, 2013.

[6] Aviation Security Manual. ICAO Doc 8973/8 